\title{
A New Fast Method of Magnetometer Calibration Based on Hexahedron Measurement
}

\author{
Yongzhu Hua ${ }^{1, a}$, Huibin Qin ${ }^{1, b}$, Bowen Luo 2, c, Jianfeng Wu $\mathbf{u}^{1, d, *}$ \\ ${ }^{1}$ School of Electronics and Information, Hangzhou Dianzi University, Hangzhou 310018, China; \\ ${ }^{2}$ Ningbo Jiangbei Yongjiang Electrical Instrument Plastic Hardware Co., Ltd, Ningbo 315000, China; \\ a229426897@qq.com, b595694779@qq.com, c1198570260@qq.com, d25791315@qq.com
}

Keywords: film resistance; thermal equilibrium equation; bernoulli equation; flow rate; wind pressure; schmidt compensation.

\begin{abstract}
According to the thermal equilibrium equation, the resistance value of the film is converted into the air flow rate, and the flow rate is converted into the wind pressure by the Bernoulli equation. The wind pressure is detected based on the film resistance. Using the digital filter to remove the collected anomaly data, combined with Schmidt compensation to remove the sensor detection process delay. Test surface based on the film resistance of the wind pressure detection system to detect the air flow and wind pressure, can be widely used in industrial, military.
\end{abstract}

\section{Introduction}

The wind pressure is the impact pressure generated by the air flow in the direction of the shock wave in the direction of its motion which can be characterized by the kinetic energy of the air in the unit volume [1]. The unit is $\mathrm{Pa}$. The wind pressure can cause the target object to deform, shift and throw and cause damage to the target. When the static pressure of the shock wave front is $5^{*} 104 \mathrm{~Pa}$, the movement speed of the air can reach $100 \mathrm{~m} / \mathrm{s}$, and the wind pressure can throw the people on the ground several meters away.

Wind pressure measurement technology, especially the blast field pressure measurement is very important in the field of military defense research. In addition, wind pressure measurement technology has been widely used in engineering protection, aviation flight and high-speed railway construction [2]. "Pitot" dynamic pressure measurement technology is mainly used to realize the pressure measurement, but in supersonic flow, the pitot head will produce in vitro shock, unable to accurately measure to flow pressure, and the lumen effect will be severely limited in its dynamic characteristics. The dynamic pressure probe method can be used to measure the wind pressure in the nuclear explosion test, but the difference between the detonation test and the explosion environment of the conventional explosion test is large, and the dynamic characteristics of the sensor are not high.

At present, the research on the theory and measurement of shock wave overpressure has obtained good results in China Domestic scholars such as Shiming Li , Bao Cui , Teng Wu, Zhongqi Wang, Jiagui Li[10]-[14]attenuation rule of shock wave overpressure in their respective works, distribution and explosion effect calculation are made in detail.

Zhongqi Wang and Bao Cui did a numerical simulation study on the protection against the far-field shock wave protection by two-dimensional multi-fluid grid method and Lagrangian fluid-solid coupling algorithm. North China institute of technology Surong Dong[15] on the analysis of the damage mechanism of cloud detonation effect, puts forward the effect of cloud detonation on the effectives of the damage effect mainly depends on explosion when the peak overpressure and instantaneous pneumatic pressure amplitude and duration. The existence of superimposed wind pressure increases the damage radius of the cloud explosion effect, thus greatly enhancing the destructive power of the target.

The wind pressure signal has the characteristics of short duration, fast mutation and large disturbance, which belongs to non-stationary random signal. The normal processing method of blast wave test signal mainly includes quasi - legal and filtering method. The effective spectral range of the 
blast wave signal is wide, the frequency component is complex, and the low frequency signal which takes up the main energy range, and the high frequency signal of certain energy [16].

The fitting waveform to be obtained can only reflect the signal change trend determined by the low frequency signal, but not the signal details determined by the high frequency signal. The classical filtering method has four methods: low pass, high pass, band pass and band resistance. Always assumes that the useful signal and hope when filtering filter element is not in a band, but in fact, the two components of spectrum overlapping phenomenon, often can appear at this time simply to use classical filtering methods will not be able to get the ideal filtering effect.

The classical filtering method generally obtains the wave band range through the spectrum analysis, and USES the signal outside the band range as a useless interference signal. However, only through frequency domain analysis can't distinguish the frequency bands of various signals accurately, and even lose information. At this point, a base function with a certain time and frequency resolution is required to analyze the signal, and the wavelet transform has the ability. Vittoria Bruni et al. [17]-[21] believe that wavelet analysis has great potential in de-noising and proposes many methods for reference.

\section{Proposed Design}

\subsection{Selection of Thermistor.}

Due to their high blast temperature and air pressure transmission speed, so the choice of thermistor measuring range need to be as high as $1000{ }^{\circ} \mathrm{C}$, the resistance of film can make them more sensitive to temperature changes, platinum resistance is commonly used in the low temperature zone of temperature measuring element, it USES platinum temperature changes in the characteristics of its resistance changes accordingly to measure temperature. Due to the stable physical and chemical properties of platinum materials, platinum resistance has high stability and is an ideal temperature measuring element[3]. Platinum resistance in $0-850{ }^{\circ} \mathrm{C}$ low temperature zone present linear relationship between the linear relationship is as follows:

$$
\begin{aligned}
& \mathrm{R}_{\mathrm{t}}=\mathrm{R}_{\mathrm{o}}\left[1+\mathrm{A} * \mathrm{t}+\mathrm{B} * \mathrm{t}^{2}+\mathrm{C}(\mathrm{t}-100) * \mathrm{t}^{3}\right] \\
& \mathrm{R}_{\mathrm{o}}=100 \Omega \\
& \mathrm{A}=3.9083 * 10^{-3} \\
& \mathrm{~B}=-5.775 * 10^{-2} \\
& \mathrm{C}=-4.183 * 10^{-4}
\end{aligned}
$$

For the non-linearity presented in the high temperature region of platinum resistance, it is necessary to use its nonlinear curve to obtain the temperature value, so the film platinum resistance conforms to the wind pressure test.

\subsection{Thermistor Resistance Sampling.}

Thermistor measurement temperature needs to convert the resistance of thermistor into voltage, and $\mathrm{A} / \mathrm{D}$ sampling process is used to obtain the change of resistance by using single chip microcomputer.In the platinum resistance high-precision measurement system, the resistance of the lead wire needs to be removed.It is usually used to eliminate the resistance of platinum resistance lead wire.The four-wire connection method is shown in figure 1:

The four-wire connection method has constant current source power lead and voltage drive lead two parts. Among them, the two leads of R1 and R2 belong to the constant current source power leads, and the film resistance is connected to the constant current source.Two voltage-driven wires in R3 and $\mathrm{R} 4$ connect the voltage signal of the platinum resistor to the instrument to amplify the circuit.Four wire connection method of constant current source is separated from temperature detection circuit, ensure that even if R1 and R2 appears two lead resistance pressure drop changes will not affect the accuracy of temperature measurement, this way of lead can completely eliminate the influence of the lead resistance, mainly used for high precision temperature measurement[4]. 


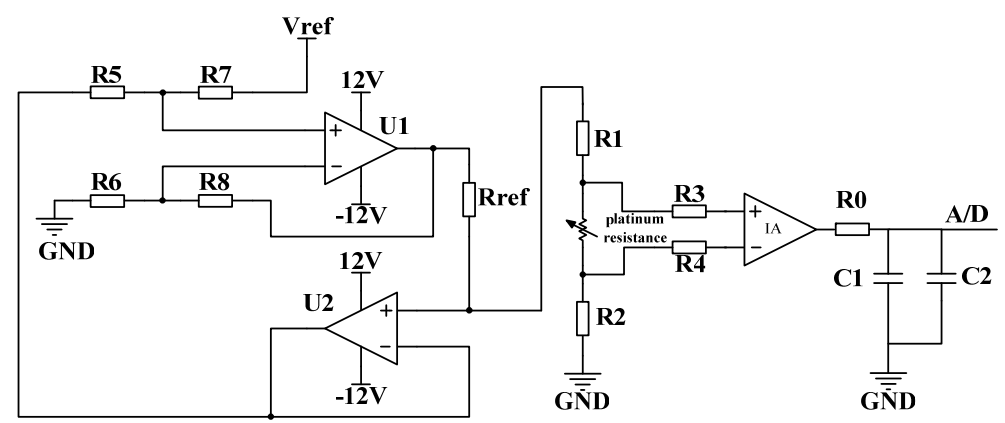

Figure 1. four-wire connection method

In order to minimize the confusion, we need to use anti-aliasing filter and sampling hold circuit to attenuate and filter the aliasing signal. The RC low pass filter circuit composed of resistance R0 and capacitor $\mathrm{C} 1$ can filter out the interference signal of the high frequency signal which is larger than the cut-off frequency of the low pass filter. The capacitance R2 is the sample holding capacitance, which can reduce the aperture error and give full play to the performance of $\mathrm{A} / \mathrm{D}$ converter, and filter the high frequency harmonics.

\subsection{Calculation of Velocity and Wind Pressure.}

The dynamic pressure has the characteristics of destructive force, so it is necessary to measure the flow rate indirectly and convert it into wind pressure. When measuring the flow rate, a stable dc current Ic is added to the film resistor to have an initial temperature. When the velocity of the air medium around the film resistance is not 0 , the temperature of the fluid medium will affect the heat of the surface of the film resistance and change the surface temperature of the film resistance. According to the thermal equilibrium equation below:

$$
\begin{aligned}
& \varepsilon=\mathrm{A}+\mathrm{BV}^{\mathrm{n}} \\
& \varepsilon=\mathrm{I}_{\mathrm{C}}^{2} * \mathrm{R}_{\mathrm{t}} /\left(\mathrm{T}-\mathrm{T}_{0}\right)
\end{aligned}
$$

A, B, n: constant of thermal parameters and other factors of the film resistance.

$\mathrm{V}$ : airflow velocity

Ic: the current of the film resistance

$\mathrm{T}$ : film resistance temperature

T0: the airflow temperature

$\varepsilon$ : heat dissipation coefficient

Rt: film resistance at the temperature $\mathrm{T}$

The Bernoulli equation can be expressed as: if the flow of the ideal fluid is constant and the influence of the fluid level is not significant, such as the flow of gas, the Bernoulli equation can be expressed as:

$\mathrm{p}+\frac{1}{2} \rho \mathrm{v}^{2}+\rho \mathrm{gh}=\mathrm{c}$

$\mathrm{P}$ : pressure ; $\rho$ :density; $\mathrm{v}$ : velocity; g: gravity acceleration; c: constant

That is, in a fluid flow, where the flow rate $\mathrm{V}$ is small, the pressure $\mathrm{P}$ is big, and where the flow rate $\mathrm{V}$ is big [5], the pressure $\mathrm{P}$ is small. We use the heat balance equation to measure the flow rate according to the film resistance, and then we can get the wind pressure according to Bernoulli equation.

\subsection{Digital Filtering Algorithm.}

Due to the volatility of the film resistance temperature characteristics, a set of resistors perpendicular to the velocity direction need to be sampled several times. The film resistance distribution is shown in figure 2 : 


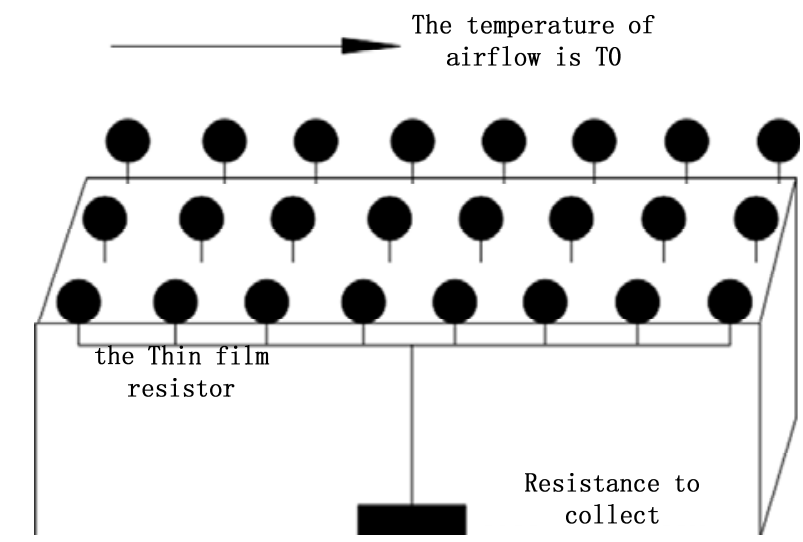

Figure 2. four-wire connection method

The results obtained by sampling need to be processed by digital filtering. Digital filtering [6] is mainly used to filter the data of a frequency band through a certain software algorithm, and finally we get a new signal. In order to further improve the filtering effect, it is sometimes possible to combine two or more digital filters with different filtering functions to form a composite digital filter, or multi-level digital filter. The filtering algorithm is combined with the arithmetic mean filtering algorithm. Specific steps are mainly first according to the actual measurement environment, first set the upper and lower limits of measurement data, and then in the process of measurement to remove more than the upper limit and lower limit, to collect data on average 10 times in a row, remove abnormal data collection process. Data comparison before and after digital filtering is shown in figure 3,4 :

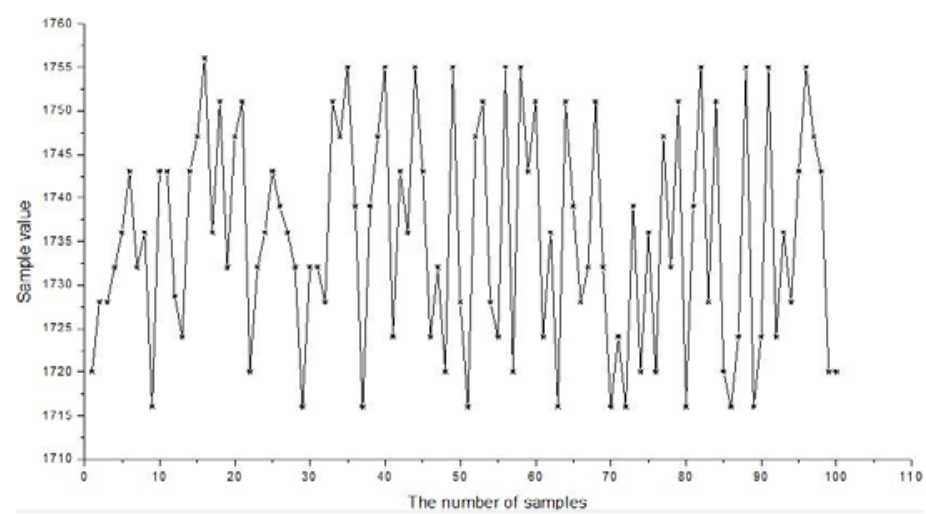

Figure 3. the sampling value before filtering

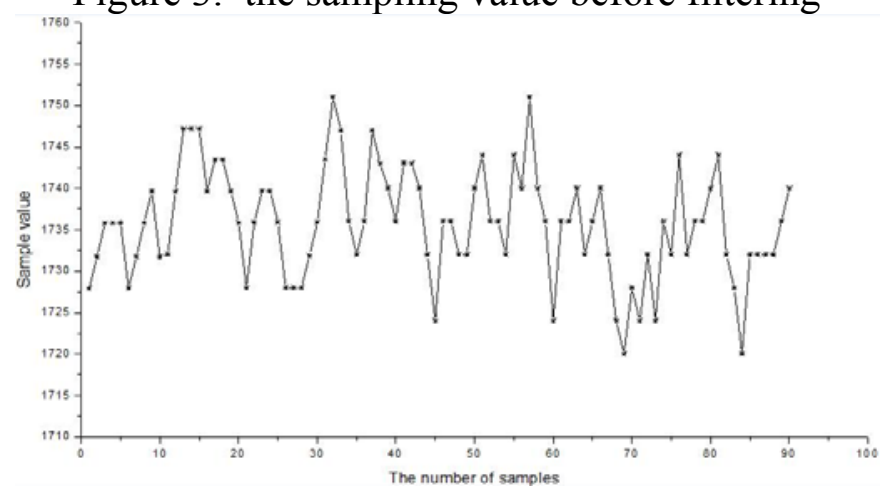

Figure 4. the sampling value after filtering

\subsection{Lag Compensation.}

In the process of measurement, there is a certain time delay in the process of resistance detection temperature and resistance resistance. The time delay will make the system unable to accurately track the input of the system. When the external disturbance disturbance system, the overshoot of the system will gradually increase. Therefore, it is necessary to compensate for the lag. In the control system, it is necessary to add an estimator on the W(s) regulator to compensate for the pure hysteresis. 
$\mathbf{G}_{\mathbf{p}}(\boldsymbol{s}) * \mathrm{e}^{-\boldsymbol{s}}$ is the control object transfer function of the system[7]. $\mathbf{G}_{\mathbf{p}}(\boldsymbol{s})$ is is a transfer function that does not contain the pure time delay characteristic in $\mathrm{G}(\mathrm{s})$.W(s) is the forward controller of the system. The principle of the Smith estimator is to parallel a compensation link at both ends of the controller W(s), which is used to compensate the delay part of the control object, which is the estimated compensator. The transfer function of Smith's compensation is shown in figure 5:

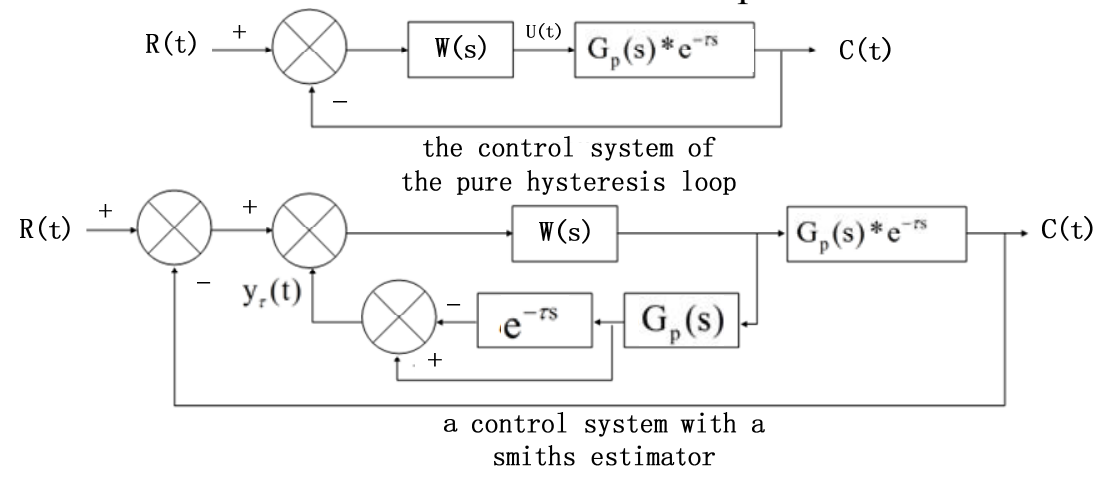

Figure 5. schmidt compensation transfer function

\section{Simulation Results}

The experimental setup is to place the pneumatic pressure detection system based on the film resistance in the explosion environment. The data collected in the experimental environment are processed and saved. According to the data collected in the end, the wind dynamic pressure waveform data is shown in figure 6:

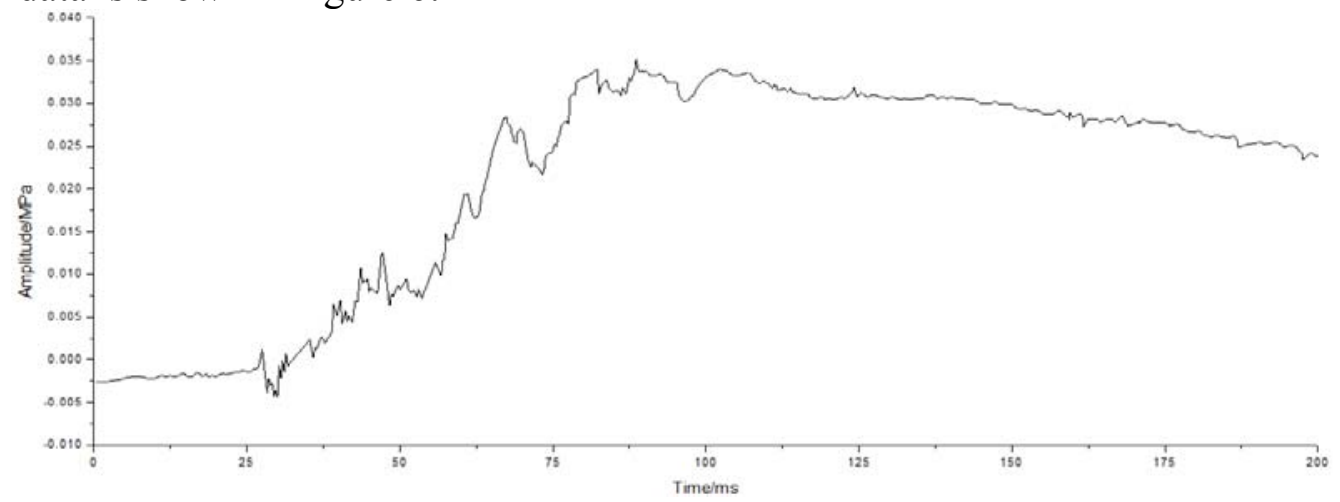

Figure 6. Wind pressure measurement waveform

\section{Summary}

In this paper, we present a system design based on thin film resistance measurement of wind pressure. This method will resistance variation according to heat balance equations will be converted to velocity, according to the Bernoulli equation to convert the velocity of air pressure, using digital filtering and lag compensation access to accurate testing data. The wind dynamic pressure detecting system based on thin film resistance can accurately detect the wind pressure, small volume, stable performance and suitable for all kinds of complicated measuring environment.

\section{References}

[1] Wang jian, li luxiang. Nuclear weapon effect and protection [M]. Beijing institute of technology press, 1993.

[2] Ji xuying. Study on wind pressure test method of explosion field [D]. Nanjing university of technology, 2015.

[3] Ren keqiang, liu hui. Digital filtering algorithm for microcomputer control system [J]. Modern electronic technology, 2003(3):15-18. 
[4] $\mathrm{Hu}$ pengcheng, weizer, mei jianting. High precision platinum resistance temperature measurement system [J]. Optical precision engineering, 2014, 22(4):988-995.

[5] Liang zhiquan. Fluid mechanics [M]. Chongqing university press, 2002.

[6] Ren keqiang, liu hui. Digital filtering algorithm for microcomputer control system [J]. Modern electronic technology, 2003(3):15-18.

[7] Ding xiaodi, research on time delay system control based on Smith estimator [D]. Jiangnan university, 2016.

[8] Roberto Alonso1 and Malcolm D. Shuster, "Attitude-Independent Magnetometer-Bias Determination: A Survey", The Journal of the Astronautical Sciences, vol. 50, no. 4, pp. 453-475 October-December 2002.

[9] Timo Pylva"na"inen, "Automatic and adaptive calibration of 3D field sensors", Applied Mathematical Modelling,vol.32, issue. 4, pages. 575-587, April 2008.

[10] Li shimin, li xiaojun, guo yanpeng. Numerical simulation of the explosion of air blast in the free field of thermal pressure explosive [J]. Blasting, 2011, 03:8.12.

[11]Cui bao, zhao jiguang, Chen jingpeng, zhang Yang. Numerical simulation of the protective effect of protective wall on far-field shockwave [J]. Journal of military engineering, sichuan, 2013, 07:104-108.

[12] Wu kaiteng. Research on three-dimensional numerical simulation of the protective effect of air shock wave on the explosion formation [J]. Journal of neijiang normal university, 2003, 02:5.11.

[13] Wang zhongqi, ning jianguo, zhao hengyang, yun shourong. Numerical simulation of the influence of retaining wall on far field explosion [J]. Explosion and impact, 2000, 01:87.91.

[14] Li jiangui, bian xiaohua, zhang lei. Comparison of numerical simulation and experimental data of explosion wave propagation [J]. Shanxi building, 2006, 08:106-107.

[15] Dong surong.Study on the damage efficiency of cloud detonation [J].Journal of north China institute of technology, 2003, 03:174-176.

[16] MAO jing, han yan. Application of wavelet analysis in blast wave signal processing [J]. Journal of missile and guidance, 2007, 04:137-138.

[17] Tangborn,A.Zhang,S.Q.Wavelet transform adapted to an approximate Kaiman filter system[J].Applied Numerical Mathematics,2000 , 33:.307-316.

[18]Lexander, M., E., Baumgartner,R., Summers,A.,R., Windischberger,C.,Klarhoefer, M., Moser, E., Somorjai, R., L.. A wavelet-based method for improving signal-to-noise ratio and contrast in MR images [J]. Magnetic Resonance Imaging.2000.18:169-180.

[19]Pecen,R.,Ula,S.,Timmerman,M.Modeling and simulation ofa Kalman Filter Based Control Scheme for an AC/DC Power System[J].Electrical Power and Energy Systems 2004, 26:173-189.

[20]Miyazaki, A, Kyushu Sangyo Univ. A study on the best wavelet filter bank problem in the wavelet-based image watermarking [J].Circuit Theory and Design, 2007.

[21]Postalcioglu.S,Erkan.K,Bolat.E.D. Comparison of Kalman Filter and Wavelet Filter for Denoising[C].Neural Networks and Brain.2005:95 1-954. 